\title{
UWB Sensor Nodes for Tracking of Human Motion in Medical and Rehabilitation Applications
}

\author{
Manmohan Sharma, Clive G. Parini and Akram Alomainy \\ School of Electronic Engineering and Computer Science \\ Queen Mary University of London, Mile End Road, London E1 4NS, United Kingdom \\ Email: $\{$ m.sharma; c.g.parini; a.alomainy $\} @ q m u l . a c . u k$
}

\begin{abstract}
This paper presents the experimental and investigational studies involving the use of compact ultra-wideband sensor nodes for tracking the human body motion. A standalone ultra-wideband positioning system developed by making use of commercially available impulse-radio UWB chips has been utilized for the localisation measurements. The operating principles of the technique used for position estimation and the results of various three dimensional localisation measurements undertaken using the system are provided. Details of the transmitter and receiver antennas and the UWB chip, the core components of this system have been discussed. Average localisation accuracy in the range of 1 to $5 \mathrm{~cm}$ has been achieved. The system makes use of time difference of arrival approach to estimate the 3D position of the target on-body node.
\end{abstract}

\section{Categories and Subject Descriptors}

I.4.8 [Scene Analysis]: Tracking; C.2.1 [Network Architecture and Design]: Wireless communication.

General Terms

Measurement, Performance, Experimentation.

\section{Keywords}

Localisation, Ultra-wideband sensors, On-body.

\section{INTRODUCTION}

Accurate determination of the location of wireless devices forms the basis of many new and interesting applications. The monitoring of the human body movements, body kinematics, is a growing research area in the fields of medical care and rehabilitation. Microwave-based localisation systems could be used to track and determine the location of wireless mobile tags. Ultra-wideband (UWB) signals can enable such positioning, especially in wireless body area networks [1]. The tags could be attached to moving objects or the human body to locate their position in real time. The mobile tags then transmit wireless signals to receiver devices in the vicinity, called readers or base stations, which process these received signals to resolve the location of the tags [2]. UWB is an extremely attractive technology for application in the field of body-centric wireless communications. UWB signals do not cause significant interference to other systems operating in the vicinity due to their low transmission power and do not pose a threat to users' safety as the radiation is non-ionizing and very low power. Typical parameters that are used in microwave-based localisation systems are time of arrival (TOA), time difference of arrival (TDOA), angle of arrival (AOA) and received signal strength (RSS). The range-based schemes such as TOA and TDOA have been shown to deliver the best localisation accuracy because of the excellent time resolution of UWB signals [3], [4].

In this paper, an experimental analysis for tracking human motion by utilizing ultra-wideband sensors has been provided. Time difference of arrival technique has been utilized to locate the position of a body-mounted UWB sensor node by making use of several base station receiver nodes. The ultra-wideband localisation system presented in this paper acts as a technology demonstrator to highlight the potential of creating a simple and inexpensive standalone system using UWB technology for locating the position of a body-mounted mobile sensor node. The paper has been organized as follows. Section 2 briefly discusses about the commercial UWB chip from DecaWave, the transmitter and receiver antennas, along with the details of the operating principles of the system. Section 3 presents the results of the localisation measurements carried out using the UWB system. Lastly, the concluding remarks for the paper have been provided in Section 4.

\section{METHODOLOGY}

\subsection{Transmitter and Receiver Antennas}

The transmitter antenna used in this investigation is a miniature CPW-fed tapered-slot UWB antenna. The antenna has a Rogers RO3210 substrate with a relative permittivity of 10.2 and thickness of $1.25 \mathrm{~mm}$. The two curved side patches in the antenna design act as the ground and let the transition of impedance to occur smoothly. Further details about this antenna can be found in a previous paper [5]. A fabricated prototype of the miniature UWB antenna has been illustrated in Figure 1(a). A wideband 
Vivaldi antenna has been used as the base station receiver, along with the miniature UWB antenna as the mobile transmitter for the localisation experiments. The Vivaldi antenna has an FR-4 substrate with a relative permittivity of 4.3 and thickness of 1.6 $\mathrm{mm}$. More details about the structure and operation of the Vivaldi antenna can be found in [6].

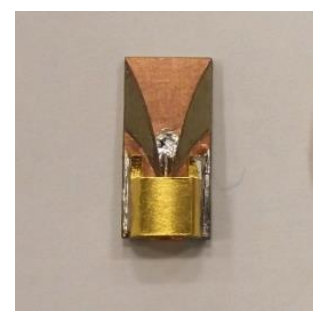

(a)

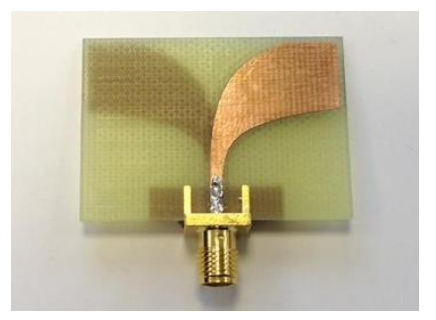

(b)
Figure 1: (a) The miniature UWB antenna and (b) the Vivaldi antenna used for the transmitter and receiver nodes respectively.

\subsection{DecaWave Chip}

The DW1000 ScenSor from DecaWave is a fully integrated low-power single chip UWB transceiver IC, compliant to the IEEE 802.15.4-2011 standard [7]. It is optimized for applications in real time location systems and wireless sensor networks across a variety of applications. It can support communication at high data rates of up to $6.8 \mathrm{Mbps}$. The chip has an excellent communication range of $300 \mathrm{~m}$ in line-of-sight (LOS) and $35 \mathrm{~m}$ in non-line-of-sight (NLOS) case. It enables location of objects using either the time of arrival or time difference of arrival schemes [8].
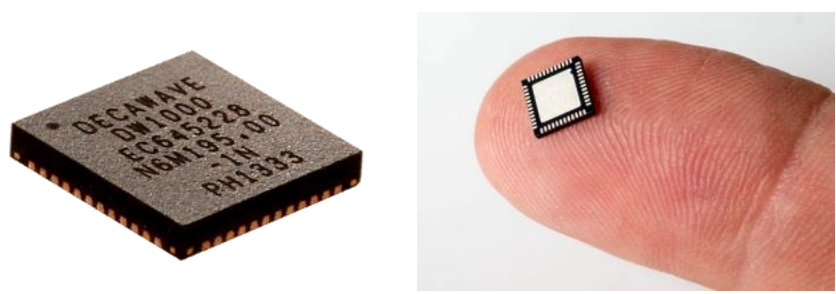

Figure 2: DecaWave's DW1000 location and communication chip [9].

The DW1000 chip supports six frequency bands with center frequencies from $3.5 \mathrm{GHz}$ to $6.5 \mathrm{GHz}$. It has a maximum receive bandwidth of $900 \mathrm{MHz}$. Also, it is very immune to multipath fading and permits reliable communication even in high fading scenarios. These capabilities of the chip to effectively handle severe multipath setting make it very suitable for utilization in reflective RF environments. Its low power consumption allows the chip to be operated from a battery for prolonged periods depending upon mode and helps in minimizing system lifetime costs. The chip has a very low silicon area which allows the implementation of cost-effective solutions for the localisation system and also helps in keeping the sensor node compact in size.

The receivers in the localisation system comprised of the ultra-wideband Vivaldi antenna integrated with the DecaWave DW1000 chip onto a PCB, as an expansion board to implement the base station sensor nodes. The mobile transmitter tag in the localisation system comprised of the miniature UWB antenna integrated with DecaWave's EVB1000 board. The EVB1000 is an evaluation board for the DW1000 IC chip, which can be utilized for the development of applications in real time location systems and wireless sensor networks [10]. The evaluation board supports all the features of the DW1000 chip. The evaluation board could be configured by means of dipswitch settings to work as a transmitter and controlled by a PC through a USB connection.

\subsection{Localisation Method}

For localisation using TDOA approach, the mobile tag transmits signals periodically and this signal is received by all the base station nodes in the vicinity. All the base station nodes need to have synchronized clocks so that the arrival time of the transmitted signal from the mobile tag to each base station could be compared [11]. For every pair of base stations, the time difference between the arrival of the signal from the mobile transmitter defines a hyperbolic surface on which the transmitting mobile tag must lie. The TDOA mechanism is beneficial for realization of a low power localisation system, as the mobile tag needs to send only a single message in order for it to be localised. Due to lesser number of messages involved, TDOA scheme is a simpler and low power solution as compared to the TOA scheme and is also suitable for applications with high tag density.

One way to obtain a TDOA measurement is to estimate TOA at each reference node and then obtain the difference between the two estimates. Since the target node and the reference nodes are not synchronized, the TOA estimates at the reference nodes include a timing offset in addition to the time-of-flight. As the reference nodes are synchronized, the timing offset is the same for each TOA estimation. This timing offset gets cancelled out when subtracting two TOA measurements [1]. Therefore, the TDOA measurement can be obtained as

$$
\hat{\tau}_{T D O A}=\hat{\tau}_{1}-\hat{\tau}_{2}
$$

where $\hat{\tau}_{1}$ and $\hat{\tau}_{2}$ denote the TOA estimates at the first and second nodes, respectively.

The TDOA associated with base station $\mathrm{BS}_{i}$ is $t_{i}-t_{1}$; i.e., it is the difference between the arrival time of the mobile station (MS) signal at $\mathrm{BS}_{i}$ and $\mathrm{BS}_{1}$. Thus, the difference between the distance values can be defined as [4]:

$$
\begin{aligned}
r_{i 1} \triangleq r_{i}-r_{1} & =\left(t_{i}-t^{0}\right) c-\left(t_{1}-t^{0}\right) c \\
& =\left(t_{i}-t_{1}\right) c
\end{aligned}
$$

It should be noted that these differences do not get affected by the errors in the MS clock time $\left(t^{0}\right)$ as it cancels out when subtracting the two time of arrival measurements.

For TDOA-based localisation, the three dimensional position of the mobile tag is obtained by the intersection of the hyperbolic surfaces defined by the TDOA values of the received signal at different pairs of base stations. Let the coordinates of the four base stations $\left(\mathrm{BS}_{1}, \mathrm{BS}_{2}, \mathrm{BS}_{3}\right.$ and $\left.\mathrm{BS}_{4}\right)$ be denoted as $\left(x_{1}, y_{1}, z_{1}\right)$, $\left(x_{2}, y_{2}, z_{2}\right), \quad\left(x_{3}, y_{3}, z_{3}\right)$ and $\left(x_{4}, y_{4}, z_{4}\right)$ respectively and $\left(x_{m}, y_{m}, z_{m}\right)$ represent the unknown coordinates of the mobile station in a Cartesian coordinate system. Without loss of generality, the origin of the Cartesian coordinate system is set at $\mathrm{BS}_{1}$, i.e., $\left(x_{1}, y_{1}, z_{1}\right)=(0,0,0)$. Let $r_{1}, r_{2}, r_{3}$ and $r_{4}$ represent the distance between the mobile station and $\mathrm{BS}_{1}, \mathrm{BS}_{2}, \mathrm{BS}_{3}$ and $\mathrm{BS}_{4}$ 
respectively. Then, using the distance formula, the following four equations could be obtained

$$
\begin{gathered}
r_{1}^{2}=x_{m}^{2}+y_{m}^{2}+z_{m}^{2} \\
r_{2}^{2}=\left(x_{2}-x_{m}\right)^{2}+\left(y_{2}-y_{m}\right)^{2}+\left(z_{2}-z_{m}\right)^{2} \\
r_{3}^{2}=\left(x_{3}-x_{m}\right)^{2}+\left(y_{3}-y_{m}\right)^{2}+\left(z_{3}-z_{m}\right)^{2} \\
r_{4}^{2}=\left(x_{4}-x_{m}\right)^{2}+\left(y_{4}-y_{m}\right)^{2}+\left(z_{4}-z_{m}\right)^{2}
\end{gathered}
$$

Equations (4), (5) and (6) can be rewritten in terms of the TDOA measurements $r_{21}, r_{31}$ and $r_{41}$ (i.e. $\mathrm{BS}_{2}-\mathrm{BS}_{1}, \mathrm{BS}_{3}-\mathrm{BS}_{1}$ and $\mathrm{BS}_{4}-\mathrm{BS}_{1}$ respectively), as

$$
\left(r_{i 1}+r_{1}\right)^{2}=K_{i}^{2}-2 x_{i} x_{m}-2 y_{i} y_{m}-2 z_{i} z_{m}+r_{1}^{2}
$$

for $i=2,3$ and 4 , where

$$
K_{i}^{2}=x_{i}^{2}+y_{i}^{2}+z_{i}^{2}
$$

By expanding and rearranging the terms, these equations can be rewritten in matrix form as

$$
\mathrm{Hx}=r_{1} \mathrm{c}+\mathrm{d}
$$

where

$$
\begin{array}{lc}
\mathrm{H}=\left[\begin{array}{lll}
x_{2} & y_{2} & z_{2} \\
x_{3} & y_{3} & z_{3} \\
x_{4} & y_{4} & z_{4}
\end{array}\right], & \mathrm{x}=\left[\begin{array}{l}
x_{m} \\
y_{m} \\
z_{m}
\end{array}\right], \\
\mathrm{c}=\left[\begin{array}{l}
-r_{21} \\
-r_{31} \\
-r_{41}
\end{array}\right], & \mathrm{d}=\frac{1}{2}\left[\begin{array}{l}
K_{2}^{2}-r_{21}^{2} \\
K_{3}^{2}-r_{31}^{2} \\
K_{4}^{2}-r_{41}^{2}
\end{array}\right]
\end{array}
$$

We can rearrange equation (9) to solve for $\mathrm{x}$ in terms of the unknown $r_{1}$, to get

$$
\mathrm{x}=\left(\mathrm{H}^{\mathrm{T}} \mathrm{H}\right)^{-1} \mathrm{H}^{\mathrm{T}}\left(r_{1} \mathrm{c}+\mathrm{d}\right)
$$

Substituting this intermediate result into (3) leads to a quadratic equation in $r_{1}$. Solving for $r_{1}$ and substituting the positive root back into (10) gives the final solution for $\mathrm{x}$.

\section{LOCALISATION MEASUREMENTS}

The UWB localisation system comprised of four base station receiver nodes aligned in a Y-shaped configuration, and a mobile transmitter node whose position is to be determined. In this system, time difference of arrival of the signal propagating from the mobile station to the four different base station receivers was used for estimating the mobile sensor node location. Use of TDOA meant that there was no need of synchronization between the mobile station and the base station receivers, as there was synchronization among the clocks of the four base stations. Thus, by using the TDOA approach, the difference between the arrival times of two signals travelling between the mobile node and the two reference nodes was estimated. For this localisation system, the distance between each base station was selected to be $50 \mathrm{~cm}$, aligned in a Y-shaped arrangement. The localisation measurements were carried out inside the Body-Centric Wireless Sensor Lab at Queen Mary University of London. For this, the UWB localisation system was placed on a table surface. In order to make the localisation system physically stable, the base station receiver nodes were fixed on to a wooden frame. This also helped to improve system deployability by reducing the overall setup time for the localisation system and ensured that the base stations remained sturdy during the entire course of the measurements.

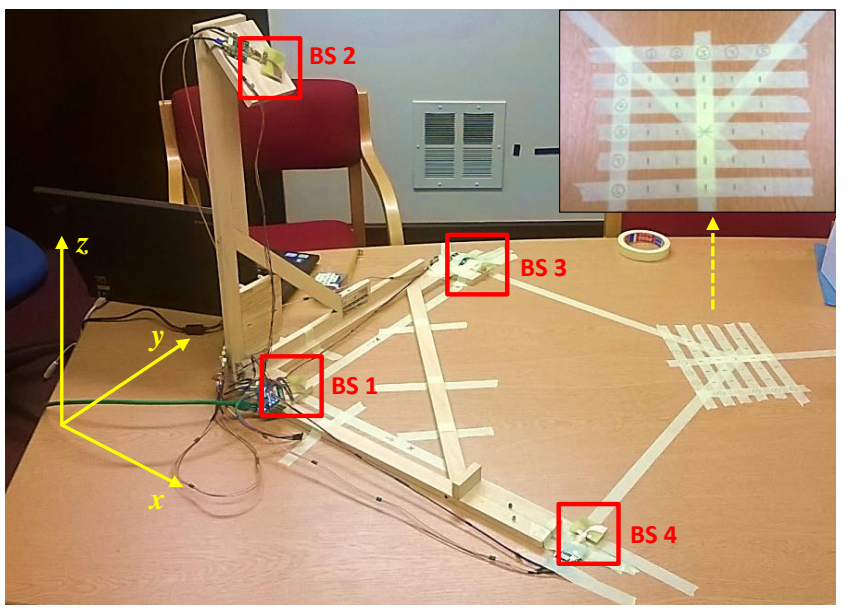

Figure 3: Measurement setup of the UWB localisation system placed on top of a table. The 5-by-5 matrix used to place the mobile station is shown in the inset.

A $50 \mathrm{~cm}$ by $50 \mathrm{~cm}$ square was drawn on the table surface, with three of the four corners of the square been occupied by the base station receivers. The base station antennas were kept about three centimeters above the table top in order to minimize the effect of the table surface on the antennas. The mobile station transmitter was placed near the fourth corner of the square. The measurements were done with the mobile station being moved on a 5-by-5 matrix near the fourth corner of the square created on the table top. Thus, the mobile station was placed at 25 different locations. The matrix had a grid spacing of $3 \mathrm{~cm}$. Figure 3 illustrates the measurement setup for the 3D localisation measurements, with the UWB localisation system fixed on top of a table and the 5-by-5 matrix locations used to place the mobile station shown in inset. The matrix point $(3,3)$ lies on the corner of the $50 \mathrm{~cm}$ by $50 \mathrm{~cm}$ square created on the table top. The measurements were done using the DW1000 chip's $900 \mathrm{MHz}$ wide frequency channel centered at $6.5 \mathrm{GHz}$. The localisation system provided the TDOA values between $\mathrm{BS}_{1}$ and each of the other three base stations, i.e., $\mathrm{BS}_{2}-\mathrm{BS}_{1}, \mathrm{BS}_{3}-\mathrm{BS}_{1}$ and $\mathrm{BS}_{4}-\mathrm{BS}_{1}$.

The localisation measurements were performed with the mobile transmitter node mounted on the hand of a real human test subject. Actual physical distances were measured from the phase centers of the mobile tag and base station antennas using a telescopic rod. From this, the $x, y, z$ coordinates were determined and the error table was constructed. A $2 \mathrm{~mm}$ thick cotton pad was placed between the on-body antenna and the skin as 'air gap'. Figure 4 illustrates the measurement setup of the localisation system, with the mobile tag attached to the test subject's hand. The hand-mounted mobile tag was then placed on each point of the 5-by-5 matrix sequentially, to cover all the 25 locations. 


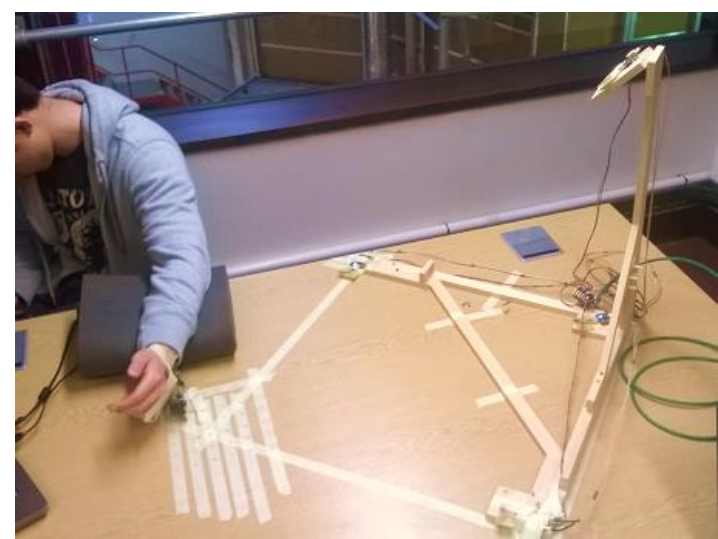

Figure 4: Localisation measurements with the mobile tag fixed on the hand of a real human test subject.

The average error values in locating the position of the body-mounted mobile tag for each row of the matrix locations have been provided in Table 1. Overall, the localisation error varied between 1 and $5 \mathrm{~cm}$ on average, when the mobile tag was mounted on the hand of a human test subject. Thus, the position estimation of the body-mounted sensor node was achieved with good level of accuracy The overall average error in estimating the $3 \mathrm{D}$ coordinates of the mobile tag for the $x, y$ and $z$ axes was 3.42 $\mathrm{cm}, 4.19 \mathrm{~cm}$ and $2.27 \mathrm{~cm}$, with a standard deviation of $2.41 \mathrm{~cm}$, $2.58 \mathrm{~cm}$ and $1.60 \mathrm{~cm}$ respectively. Some part of the localisation error could be accounted for by the subject movement as well, since it is very difficult for a human subject to remain absolutely still during course of the measurements.

Table 1: Average error in estimating the $\boldsymbol{x}, \boldsymbol{y}$ and $\boldsymbol{z}$ coordinates of the hand-mounted mobile tag for each row of the matrix locations.

\begin{tabular}{|c|c|c|c|}
\hline & \multicolumn{3}{|c|}{ Average Error (cm) } \\
\hline & $x$ & $y$ & $Z$ \\
\hline Row 1 & 4.18 & 2.92 & 0.92 \\
\hline Row 2 & 2.97 & 5.03 & 2.44 \\
\hline Row 3 & 3.88 & 3.57 & 1.89 \\
\hline Row 4 & 3.60 & 4.76 & 3.07 \\
\hline Row 5 & 2.47 & 4.71 & 3.05 \\
\hline Overall Mean & 3.42 & 4.19 & 2.27 \\
\hline Standard Deviation & 2.41 & 2.58 & 1.60 \\
\hline
\end{tabular}

\section{CONCLUSION}

In this paper, experimental studies involving the use of ultra-wideband sensor nodes for human motion tracking applications have been presented, by means of a 3D localisation system comprising of a body-mounted mobile tag to be localised and various off-body base station receivers. This standalone localisation system was able to estimate the position of the on-body mobile node with an accuracy ranging between 1 and 5 $\mathrm{cm}$. This system acts as a technology demonstrator to highlight the potential of creating a simple and inexpensive standalone system using UWB technology for tracking body-mounted mobile sensor nodes. The experimental work presented in this paper has successfully validated that the pulsed UWB technology can be easily utilized to track small movements of human limbs and thus this simple system could be easily scaled up to realize a full body motion tracking system, comprising of multiple body-mounted mobile tags. Related research has shown that improved accuracy (down to $<1 \mathrm{~cm}$ ) can be achieved by employing a wider operating bandwidth than that used by the DecaWave chip $(900 \mathrm{MHz})$, as well as using the higher frequency end of the UWB band.

\section{ACKNOWLEDGMENTS}

The authors would like to thank Dr. Massimo Candotti and Mr. Qiao Cheng of the School of Electronic Engineering and Computer Science, QMUL for their assistance with the setup and on-body measurements.

\section{REFERENCES}

[1] Z. Sahinoglu, S. Gezici, and I. Guvenc, Ultra-Wideband Positioning Systems: Theoretical Limits, Ranging Algorithms, and Protocols. Cambridge University Press, 2008.

[2] G. Villarrubia, J. Bajo, J. F. De Paz, and J. M. Corchado, "Real time positioning system using different sensors," in 16th International Conference on Information Fusion (FUSION), 2013, pp. 604-609.

[3] R. Thomä, R. H. Knöchel, J. Sachs, I. Willms, and T. Zwick, Eds., Ultra-Wideband Radio Technologies for Communications, Localization and Sensor Applications. Rijeka: InTech, 2013.

[4] A. H. Sayed, A. Tarighat, and N. Khajehnouri, "Network-based wireless location: challenges faced in developing techniques for accurate wireless location information," IEEE Signal Process. Mag., vol. 22, no. 4, pp. 24-40, Jul. 2005.

[5] M. Sharma and C. G. Parini, "A miniature wideband antenna for wearable systems," in 2013 Loughborough Antennas \& Propagation Conference (LAPC), 2013, pp. 619-623.

[6] M. Sharma, C. G. Parini, and A. Alomainy, "Experimental investigation of 3D localisation of an onbody UWB antenna using several base stations," in Loughborough Antennas and Propagation Conference (LAPC), 2014, pp. 173-177.

[7] E. Karapistoli, F.-N. Pavlidou, I. Gragopoulos, and I. Tsetsinas, "An overview of the IEEE 802.15.4a Standard," IEEE Commun. Mag., vol. 48, no. 1, pp. 4753, Jan. 2010.

[8] "DecaWave DW1000 ScenSor - User Manual," 2014. [Online].

Available:

http://www.decawave.com/sites/default/files/resources/d w1000_user_manual_2_02.pdf.

[9] "ScenSor Radio Comunication Products Overview DecaWave." [Online]. Available: http://www.decawave.com/products/overview.

[10] "Product Information: EVB1000," 2013. [Online]. Available:

http://www.decawave.com/sites/default/files/resources/ev b1000-product-brief_3.pdf.

[11] S. Gezici, "A Survey on Wireless Position Estimation," Wirel. Pers. Commun., vol. 44, no. 3, pp. 263-282, Oct. 2008. 